\title{
Premixed nitrous oxide/oxygen as an analgesic for dressing in patients with perianal abscess after surgery: study protocol for a randomized, within- patient, controlled trial
}

Jun-Jun Zhang

Ningxia Medical University

Hai-Xiang Gao

Ningxia Medical University

Ting-Ting Zhang

Ningxia Medical University

Wen-Qiang Bao

Ningxia Medical University

Jian-Ying Mou

Ningxia Medical University

Kai Meng

Ningxia Medical University

Ya-Liang Dai

Ningxia Medical University

Lu-Lu Gao

Ningxia Medical University

Qaing Liu

Ningxia Medical University

Zhi-Jing Li

Ningxia Medical University

Zhi-Ming Ge

Ningxia Medical University

Yun Yang

Ningxia Medical University

Jian-Qiang Yu

Ningxia Medical University

Yu-Xiang Li (D li_yuxiang@163.com )

Ningxia Medical University 


\section{Study protocol}

Keywords: Procedure Pain, Analgesia, Nitrous oxide, Perianal Abscess

Posted Date: August 8th, 2019

DOl: https://doi.org/10.21203/rs.2.12499/v1

License: (c) (i) This work is licensed under a Creative Commons Attribution 4.0 International License. Read Full License 


\section{Abstract}

Background:Perianal abscess is mainly treated by surgery in the clinic. However, the pain induced by dressing is the most prominent problem in postoperative patients and available analgesics is limited. The short- acting analgesia, premixed nitrous oxide/ oxygen, may be an ideal analgesic for acute pain induced by dressing change after perianal abscess surgery. This study aims to evaluate the safety and analgesic efficacy of premixed nitrous oxide/oxygen mixture for patients with dressing change after surgery around the anus. Methods/design:This protocol for a randomized, double-blind, placebocontrolled trial will be implemented in the Anorectal Department, Yinchuan Hospital of Traditional Chinese Medicine affiliated to Ningxia Medical University. Study subjects are hospitalized patients underwent perianal abscess surgery and suffered from moderate to severe pain due to dressing. Two hundred subjects will be selected and randomly assigned to other intervention or control group. The intervention group will get regular pain treatment plus premixed nitrous oxide/oxygen and the control group will be treated with regular pain treatment plus oxygen. The patients, medical staffs or investigators are blinded to the nature of the gas mixture in each cylinder and the randomization list. Data will be collected at baseline (T0, 2 min before dressing) and at 5 min (T1) after the beginning of intervention and at $5 \mathrm{~min}$ (T2) past dressing. The primary outcome will be the change in pain score at T1 and T2. Secondary outcomes cover physiological parameters, adverse events, patients and medical staffs' satisfaction as well as patients' acceptance. Discussion:The findings of this trial will provide a valuable therapeutic regimen for pain caused by dressing after perianal abscess surgery and identify the efficacy and safety of the gas.

\section{Background}

Perianal abscess, a collection of infected fluid under the skin around the anus, is an acute suppurative disease of soft tissue caused by bacteria [1]. Its incidence is difficult to accurately count because there are many uncertainties. A previous study showed that anorectal diseases account for about $15 \%$ of all surgical diseases, and the incidence of perianal abscess was $0.4 \%$ [2]. It's reported that $90 \%$ of cases are caused by anal gland infection[1,3]. As of now, surgery is the main method for the treatment of perianal abscess [4].

Painful dressing are frequently required during the treatment of wounds. Patients may suffer the severe pain caused by reduplicative dressing change. The pain caused by dressing is the most prominent clinical problem in patients with perianal abscess [5]. Uncontrolled dressing pain may induce anxiety and reluctance to participate in their wound care, thus lead to the delayed recovery and condition to deteriorate. The influence of pain on patients is not restricted to prolong hospitalization, urinary retention, defecate difficulty, and other complications, but is also evident among pathophysiological changes, such as emotional tension, increased heart rate, and elevated blood pressure [5-7]. Postoperative dressings play an important role in the overall process of anorectal treatment. The successful dressing or not is directly related to the incidence of wound infection, the length of the and the degree of wound healing [8]. 
Although there are many analgesic methods for postoperative dressings, the efficacy is not ideal. Local injection of analgesics (such as intravenous morphine, the onset of action is 3 minutes and the blood concentration peaks after 10 minutes, is an effective analgesic treatment for acute pain [9]) can relieve pain in time. But in most cases, it is not recommended due to invasive administration. As for oral opioids, studies $[10,11]$ have shown that medical staffs and patients still have many misconceptions about its addiction, tolerance and toxic side effects like acute respiratory depression, ulcers, bleeding, which give rise to the insufficient use of analgesia. In China, the situation is even more pronounced. There is a statistical statement that China ranks 83rd (among 179 countries list) in per capita opioid consumption, far behind some small countries and regions [12].

The premixed nitrous oxide/oxygen stored in the cylinder is a self-service inhaled gas. The inhalation system consists of a whistle, mask and breathing valve that is one-way. Its analgesic effects have been known for nearly two centuries. Studies [13-15] have confirmed the safety of premixednitrous oxide used pain management. In addition, the main advantage of this analgesic gas is that it can't cause loss of consciousness, and thus patients can control their own maintenance of gas flow [16]. In addition, there is few side effects due to its poorly blood/gas solubility and bound to proteins as well as characterized by rapid onset (1-2 minutes) and transient effects ( $<1 \mathrm{~min})$ after withdrawal $[17,18]$. Even if it occurs, usually, it disappears quickly after exposure to air [16]. There are no reports of the use of nitrous oxide/oxygen mixture to relieve pain caused by dressing after perianal abscess surgery yet. So, we hypothesize that the premixed nitrous oxide/oxygen is a optional, safe and effective gas as an analgesic for dressing after perianal abscess surgery. We designed this study protocol to test and verify this hypothesis.

\section{Methods}

\section{Study design}

This study protocol is a randomized, within-patient, controlled, double-blind, single- center trial (See Fig. 1). This clinical trial was designed on the basis of interventional Trials SPIRIT [19] guidelines (See Additional file 1) as well as abiding by the checklist set out in the Consolidated Standards of Reporting Trials (CONSORT 2010) [20]statement (see Additional file 2). The study have obtained an official authorization from the Ethics Committee of Ningxia Medical University (2018-231) and was registered in the China Clinical Trial Registration Center (NO.ChiCTR1900021374) for the clinical trial protocol. We will summarize the complete schedule of participant enrollment, intervention, and evaluation in table 1.

\section{Study setting}

This study will be conducted by a trained researcher in the Anorectal Department, Yinchuan Hospital of Traditional Chinese Medicine affiliated to Ningxia Medical University. The department is the only nationallevel anorectal specialty in our district, with visiting more than 6,000 outpatient and inpatient 
approximately 1200 people annually. Hence, we have sufficient patient to achieve a suitable and crosssectional sample size.

\section{Study population}

According to the study design, a total of 200 eligible participants will be recruited from 28 April 2019 in the Anorectal Department. To eliminate the complex factors that may interfere with dressing, we will recruit patients with relatively consistent or close of wound conditions as far as possible.

\section{Inclusion criteria}

- Chinese-speaking and ranging from 18 to 60 years old;

- Meeting the diagnosis of perianal abscess [21] and are suffering from acute pain (moderate to severe intensity) induced by dressing after perianal abscess surgery.

- Being able to take deep breaths to use use self-managing devices (similar to oxygen mask);

- Signing the informed consent.

\section{Exclusion criteria}

- Having contraindications to the use of nitrous oxide/oxygen (such as intracranial hypertension, maxillofacial traumatism, gas embolism, unventilated gas in the body, requiring pure oxygen inhalation) [22];

- Impairing cognitive function and being difficult to report pain;

- Being diagnosed with other life-threatening situations or instability of vital signs.

\section{Randomization, allocation concealment, and blinding}

A total of 200 participants will be randomly assigned to either intervention or control group in a 1:1 fashion with a computer-generated list. Taking into account selection bias and confounding factors, the random numbers of each participant will be generated by an independent statistical expert who works for the School of Public Health and Management, Ningxia Medical University with applying Random Allocation Software version 1.0.0 [23]. The expert will not be involved in the recruitment, gas distribution, date collection. Then, the random numbers will be stored and sealed in a designated research office to ensure concealment [15]. In order to maintain a double-blind environment, nurses and researchers are blinded and not allowed to touch the random numbers unless they can put forward justifiable reason and get the approval from the project supervisor. Only the project leader who is in charge of gas distribution 
and treatment delivery can have access to the envelope [23]. In addition, the two gas cylinders (Ningfeng Oxygen Company, Yinchuan, China) containing premixed nitrous oxide/oxygen and oxygen are strictly identical on the package, and all cylinders are marked with the letters A or B. The intervention group will receive premixed nitrous oxide/oxygen contained in gas cylinders tag the letter $A$. While the control group will obtain oxygen stored in cylinders with $B$. No medical staffs, researchers and patients know what the letter indicates. The treatment method (the nature of the gas inhaled by patients) is blind for everyone. According to study rules of the protocol, the investigators will know the answer until completing all the trials.

\section{Interventions}

To build the same conditions, dressing for all post-operative wounds is performed by a regular surgeon who has received medicine college education for 5 years or longer and more than 3 years of clinical experience in the Anorectal Department. The patients will receive an informed consent form and get an explanation from investigators regarding the aim, benefits and possible risks of the trial before the study is carried out. Eligible patients will be randomly allocated into two groups, inhaling premixed nitrous oxide/oxygen or oxygen with a one-way valve, specially designed oral-nasal mask [15]. The patients hold the self-administered mask that can be controlled by their own negative pressure to inhale gas until the dressing is over. Regular pain treatment will also be conducted as usual. In this study, we designed that the intervention group will get nitrous oxide/oxygen plus conventional analgesic therapy comparing with the control group receives oxygen plus conventional analgesic therapy. In general, inhalation of these two gases is a safe intervention strategy with no severe adverse effects on patients. Even if any undesired events during the nitrous oxide/oxygen intervention happen, short and mild, which usually disappear when the patients are exposed to the air [16]. Finally, each participant is endowed with undeniable right to withdraw from the study at any time, of course, the reason of dropout will also be recorded and analyzed by the Data Monitoring Committee (DMC) and the data collector.

\section{Outcome measurement}

\section{Baseline data}

Demographic details of each patient (age, sex, nationality, weight, height, education level, occupation, etc.) and clinical characteristics (such as classification of perianal abscesses and post-operation wound size) will be recorded using in-house-designed questionnaire at baseline (T0). Physiological parameters including blood pressure, heart rate, respiratory rate, oxygen saturation and temperature will also be collected.

2. Primary outcome measure 
The primary outcome is the change in pain score assessed on the Changhai Pain Rating Scale (it combines the characteristics of the Faces Pain Scale Revised, Numerical Rating Scale and Visual Analogue Scale). The researchers will measure and record the primary measure with the patients selfreport scale at T0 (3 min before dressing), T1 (5 min after the beginning of dressing) and T2 (5 min after dressing finished) respectively. The percentage change in pain score at $\mathrm{T} 1$ and $\mathrm{T} 2$ will be then calculated.

\section{Secondary outcome measures}

Secondary outcomes measures cover physiological parameters, adverse events, patients and medical staffs' satisfaction, Ramsay sedation score and patients' acceptance. Noninvasive electronic manometer (OMRON, HEM-7120) and digital monitoring of oxygen saturation (OXIMETER, PC-60B) will be used to monitor the physiological parameters. And these parameters will be recorded by data collector at T0, T1 and T2. However, adverse events in secondary outcome measures, such as nausea, vomiting, numbness, drowsiness, over sedation and dizziness [14] will be carefully recorded after performing inhalation gas and handled properly. Patients and medical staffs' satisfaction (a five-point satisfaction scale, $1=$ very dissatisfied; 5 = very satisfied) with pain management will be obtained at T2. Also, acceptance from the patients (they are asked if they would still accept this new analgesic gas in case of acute pain with moderate to severe, yes / no) will be recorded at $5 \mathrm{~min}$ after the whole intervention. Finally, total time of the patient inhales gas will be also minuted.

\section{Sample size determination}

The determination of its sample size was on the basis of preliminary experimental data collected in the Anorectal Department for 30 patients. and our previous studies involved burn-dressing and cancer breakthrough pain $[15,24]$. The data indicated that $55 \%$ of participants receiving premixed nitrous oxide/oxygen had a meaningful change in pain score (a drop at least one-third of pain score [25]) at T1 and $\mathrm{T} 2$, while $15 \%$ of patients receiving oxygen was. That is, we designed the intensity of pain as the primary outcome of this trial, so when the difference between the two groups is the most obvious and decimal in two places, the pilot experiments showed that the mean \pm standard deviation of the control group is $7.67 \pm 0.67$, while the experimental group is $4.47 \pm 0.86$. To assess the efficacy of the new analgesic gas, statistical power of $90 \%(\beta=0.10)$ and type- 1 error rate of 0.05 (a two-tail test) is needed. So a sample size of 87 is sufficient. Considering a possible $15 \%$ dropout rate, ultimately, we decided that 200 patients in total will be recruited to meet the Chinese Food and Drug Administration standard.

\section{Data management}

In order to ensure the quality of the collected data and the smooth implementation of the study protocol, all the researchers will obtain professional and targeted training concerning aim, design (randomized and double-blind), data collection and the management of adverse effects as well as withdrawal records and analysis involved in the trail before the study [23]. Not just have the DMC and auditing system been set 
up to monitor data collection and patients safety throughout the study, but double check method of data has been established for quality control. The monitors and auditors are responsible for checking this password-protected database periodically, but they are blind to random allocation. They are not involved in research design, data collection and management, manuscript writing and publication, etc. Microsoft Office 2010 (Redmond, WA, USA) will be elected to input data, this is the responsibility of the data collector [23]. During the database established, anonymity runs the study and personal information (name, number, etc.) of participants are kept confidentiality in other locked cabinet. In other words, all data involved in the study will be entered by two people independent of the protocol through double password entry. Only when the final statistical analysis is performed, the researchers can be access to the database. If there is missing data, a sensitivity analysis is necessary. All original records will be kept at least 3 years, both electronic and paper.

\section{Data analysis}

The statistic analysis of the data will be conducted by a statistician with SPSS version 22.0 (Chicago, IL, USA) in the light of the CONSORT statement [20]for guiding control randomized trials (CRTs). Multiple imputation and/or last observation carried forward (LOCF) will be used to handle the missing data. Descriptive statistics (such as population demographic data) will be performed by medians (inter-quartile ranges, IQR), means (standard deviations, SD), and proportions (exact binomial 95\% confidence interval, $\mathrm{Cl}$ ). Comparison of mean between two groups will be analyzed by $t$ test (normal distribution, homogeneity of variance) or Mann-Whitney test (non-normal distribution, two-sample), while categorical variables (comparison of proportions for the two groups) will be assessed by Chi-squared tests or Fisher' s exact test.When $P$ values (two-sided) is less than 0.05 , we will consider that there is significantly statistical.

\section{Discussion}

"Access to pain management is a fundamental human right", as the Declaration Montreal in International Pain Summit at 2010 announced [26].It is an obligation for all medical staff to consider making the treatment resources reasonably available to relieve pain within their own professional practice scope. However, many studies $[10,11]$ indicates that health care professionals are reluctant to offer adequate pain management for people with pain (moderate or severe) due to adverse effects of morphine. In addition, they are lack of knowledge about the mechanisms and management of pain [26], so there is a tendency for healthcare professionals to focus on the underlying pathology but ignore pain [27].Moreover, there is very strict control over the use of opioids. The medical consumption per capita ranks the bottom of The International Narcotics Control Board (INCB) list [12].Traditional Chinese culture encourages people to be brave by enduring the pain [28].These lead to inadequate pain management. However, study also shows that some technologies can reduce pain to tolerable levels, even in low-resource settings [27]. In fact, existing researches have revealed that not just can mixture of nitrous oxide/oxygen as an analgesic descend opioids consumption $[29,30]$, but it is safety and effective in the process of pain 
management $[14,24]$. Of course, there is report that inhalation of nitrous oxide can't show significant analgesic effectiveness for advanced cancer patients with breakthrough pain [31].

The planned trial aims to identify the the clinical safety and analgesic efficacy of the nitrous oxide/oxygen mixture on patients with acute pain induced by dressing. If the finding of this study has an active effect on dressing induced pain, the result could help to generate preliminary pain guidelines in patients with acute pain and improve patients' overall satisfaction with the Anorectal Department in China.We be firmly convinced that the result may be disseminated to domestic and overseas journals and conferences.

\section{Trial status}

Currently, patient recruitment is ongoing.Trial registration: ChiCTR1900021374. Registered on 17 February 2019. Eligible participants will be recruited from 28 April to 28 December 2019 in the Anorectal Department.

\section{Abbreviations}

CONSORT: Consolidated Standards of Reporting Trials; CRTs: control randomized trials; DMC:Data Monitoring Committee; INCB: International Narcotics Control Board; LOCF:last observation carried forward; SPIRIT:Standard Protocol Items for Randomized Trials.

\section{Declarations}

\section{Ethics approval and consent to participate}

This study has obtained ethical approval from the Ningxia Medical University Ethics Committee (2018231). Patients voluntarily signed a conformed consent to participate, prior to the study. We tend to disseminate international journals and conferences.

\section{Consent for publication}

Not applicable.

\section{Availability of data and materials}

Not applicable. 


\section{Competing interests}

Authors declare that there is no conflict of interest.

\section{Funding Statement}

This study received support from the Science and Technology Support Program (grant number: 2018CMG03005) and the Key Research and Development Project (project number: 2019BEG03058) of Ningxia.

\section{Acknowledgements}

The patients and staff at Anorectal Department, Yinchuan Hospital of Traditional Chinese Medicine, is gratefully acknowledged.

\section{Author contributions}

Study concept and design: Dr. Y-X L, J-Q Y; Acquisition of subjects and/or data: MS. H-X G, K M, Y-L D, Y Y, Z-J L and Z-M G; Analysis and interpretation of data: Dr. L-L G, Q Land MS. W-Q B, J-Y M; Preparation of manuscript: Dr. Y-X L and MS. J-J Z and T-T Z.

\section{References}

1. Kapil S., Samuel O A., Phillip J T., Josef W., Robin K P. Perianal abscess. BMJ Open 2017;356:1-8.

2. Shrum RC. Anorectal pathology in 1000 consecutive patients with suspected surgical disorders. Dis Colon Rectum 1959;2:469-72. [PMID: 14446233]

3. Ommer A., Herold A., Berg E et al. Cryptoglandular anal fistulas. Dtsch Arztebl Int 2011;108:707-13.

4. Stella R S., Katy N., Jennifer A S., Jo C D., Zipporah I. E., Lyndsay E P., Paul J B., et al. Internal dressings for healing perianal abscess cavities. Cochrane Database of Systematic Reviews 2016;8:1-41.

5. Li Xu, Yu-Ti Yin, Wen-Hui Fang et al. Efficacy evaluation of TCM sequential therapy for relieving pain change after anorectal disease. Chinese Journal of Basic Medicine in Traditional Chinese Medicine 2014;20(6):804-6.

6. You-Qing Huang. Mechanism and treatment of postoperative pain in anorectal diseases. Asia-Pacific Traditional Medicine 2011;7(5):102-3.

7. Fink WA. The pathophysiology of acute pain. Emerg Med Clin North Am 2005; 23(2):277-84. 
8. Xiu-Xiu Gao, Xiu-Mei. Observation on the effect of pain nursing on dressing change after anorectal disease operation. Proceeding of Clinical Medicine 2012;21(3):230-1.

9. Mercadante S, Villari P, Ferrera P, Bianchi M, Casuccio A. Safety and effectiveness of intravenous morphine for episodic (breakthrough) pain using a fixed ratio with the oral daily morphine dose. $\mathrm{J}$ Pain Symptom Manag 2004; 27(4):352-9.

10. Yuxiang L, Lingjun Z, Lu T, Mengjie L, Xing M, Fengping S, et al. Burn Patients' experience of pain management: a qualitative study. Burns 2012;38(2): 180-6.

11. Kariman H, Majidi A, Amini A, Dolatabadi AA, Derakhshanfar $H$, Hatamabadi $H$, et al. Nitrous oxide/oxygen compared with fentanyl in reducing pain among adults with isolated extremity trauma: a randomized trial. Emerg Med Australas 2011;23(6):761-8.

12. International Narcotics Control Board (INCB). [cited 2015]. Available from: https://idpc.net/policyadvocacy/global-advocacy/global-drug-control-system/international-narcotics-control-board

13. Myles PS, Leslie K, Chan MTV, et al. The safety of addition of nitrous oxide to general anaesthesia in at-risk patients having major non-cardiac surgery (ENIGMA-II): a randomised, single-blind trial. Lancet 2014;384:1446-54.

14. Li YX, Tang L, Yu JQ, Dai XY, Zhou WF, Zhang WN, et al. Analgesia effect of a fixed nitrous oxide/oxygen mixture on burn dressing pain: study protocol for a randomized controlled trial. Trials 2012;13:67.

15. Liu Q, Wang Y, Luo XJ, Wang NJ, Chen P, Jin X, et al. A fixed inhaled nitrous oxide/oxygen mixture as an analgesic for adult cancer patients with breakthrough pain: study protocol for a randomized controlled trial. Trials 2017;18(1):13.

16. Maslekar S, Gardiner A, Hughes M, Culbert B, Duthie GS. Randomized clinical trial of Entonox versus midazolam-fentanyl sedation for colonoscopy. Br J Surg 2009;96:361-8.

17. Maslekar S, Balaji P, Gardiner A, Culbert B, Monson JR, Duthie GS. Randomized controlled trial of patient controlled sedation forcolonoscopy: entonox versus modified patient maintained target controlled propofol. Colorectal Dis 2011;13 (1):48-57.

18. Maslekar S, Gardiner A, Hughes M, Culbert B, Duthie GS. Randomized clinical trial of Entonox ${ }^{\circledR}$ versus midazolam-fentanyl sedation for colonoscopy. Br J Surg 2009;96:361-8.

19. Chan AW, Tetzlaff JM, Altman DG, Laupacis A, Gøtzsche PC, Krleža-Jerić K, et al. SPIRIT 2013 statement: defining standard protocol items for clinical trials. Ann Intern Med 2013;158:200-7.

20. Kiriakou J, Pandis N, Madianos P, Polychronopoulou A. Assessing the reporting quality in abstracts of randomized controlled trials in leading journals of oral implantology. J Evid Based Dent Pract 2014;14(1):9-15.

21. China Association for Traditional Chinese Medicine. Guidelines for Diagnosis and Treatment of Commom Diseases of Coloproctology in Traditional Chinese Medicine. 1th ed, revised. Beijing: Chinese Press of Traditional Chinese Medicine, 2012:1-7.

22. X. Moisset, M. A. Sia, B. Pereira, F. Taithe, E. Dumont, L. Bernard, P. Clavelou. Fixed 50:50 mixture of nitrous oxide and oxygen to reduce lumbar puncture- induced pain: a randomized controlled trial. 
European Journal of Neurology 2016; 24(1):46-52.

23. Lu-Lu Gao, Li-Shan Yang, Jun-Jun Zhang, Yi-Ling Wang, Ke Feng, Lei Ma, et al. A fixed nitrous oxide/oxygen mixture as an analgesic for trauma patients in emergency department: study protocol for a randomized, controlled trial. Trials 2018;19:527.

24. Li YX, Han WJ, Tang HT, Wu YS, Tang L, Yu J Q, et al. Nitrous oxide-oxygen mixture during burn wound dressing: a double-blind randomized controlled study. CNS Neurosci Ther 2013;19(4):278-9.

25. Salas S, Auquier P, Duffaud F, Garnier SR, Deschamps M, Honoré S, et al. Efficacy of lidocaine in patients receiving palliative care with opioid-refractory cancer pain with a neuropathic component: study protocol for a randomized controlled study. Trials 2014;15:318.

26. Cousins MJ, Lynch ME. The Declaration Montreal: access to pain management is a fundamental human right. Pain 2011;152(12):2673-4.

27. Aisuodionoe-Shadrach $O$. Acute trauma and preoperative pain. In: Kopf A, Patel NB, editors. Guide to pain Management in low-Resource Settings. Seattle: IASP;2010; 115-8.

28. Qiang Liu,Yu xiang Li et.al. Breakthrough Pain: A Qualitative Study of Patients with Advanced Cancer in Northwest China. Pain Management Nursing 2018;19(5):506- 15.

29. Kariman H, Majidi A, Amini A, Dolatabadi AA, Derakhshanfar $H$, Hatamabadi $H$, et al. Nitrous oxide/oxygen compared with fentanyl in reducing pain among adults with isolated extremity trauma: a randomized trial. Emerg Med Australas 2011;23(6):761-8.

30. Abdelkefı A, Abdennebi YB, Mellouli F, Othman TB, Torjman L, Ladeb S, et al. Effectiveness of fixed $50 \%$ nitrous oxide oxygen mixture and EMLA cream for insertion of central venous catheters in children. Pediatr Blood Cancer 2004;43 (7):777-9.

31. Enting RH, Oldenmenger $\mathrm{WH}$, van der Rijt CC, Koper P, Lieverse J, Smitt PA. Nitrous oxide is not beneficial for breakthrough cancer pain. Palliat Med 2002;16(3):257-9.

\section{Table 1}

Table 1 Schedule of enrolment, interventions, and assessments. 
Time points Study period

\begin{tabular}{lllll} 
Enrolment & $\begin{array}{l}\text { Post- } \\
\text { allocation }\end{array}$ & & Close-out \\
\hline $\begin{array}{l}\text { suffering from acute pain induced by } \\
\text { dressing after surgery }\end{array}$ & T0 & T1 & T2 & $\begin{array}{l}\text { Data collection } \\
\text { measurements }\end{array}$
\end{tabular}

\begin{tabular}{ll} 
Patients & \\
$\begin{array}{l}\text { Eligibility } \\
\text { screen }\end{array}$ & $\sqrt{ }$ \\
$\begin{array}{l}\text { Informed } \\
\text { consent }\end{array}$ & $\sqrt{ }$ \\
\hline
\end{tabular}

Allocation

$\sqrt{ }$

Interventions

Control

group

Treatment

group

\section{Assessments}

Pain score

$\begin{array}{llllll}\sqrt{ } & \sqrt{ } & \sqrt{ } & \text { Changhai Pain }\end{array}$

Rating Scale

Blood

pressure

$\sqrt{\sqrt{ }} \quad \sqrt{ } \quad \sqrt{ } \quad$ Noninvasive

electronic

manometer

Heart rate

$\begin{array}{lllll}\sqrt{ } & \sqrt{ } & \sqrt{ } & \text { Noninvasive }\end{array}$

electronic

manometer

Oxygen

saturation

$\begin{array}{lllll}\sqrt{ } & & \sqrt{ } & \sqrt{ } & \text { Digital } \\ & & & & \end{array}$

Side effect

$\sqrt{ } \quad \sqrt{ } \quad$ Yes_/no

Acceptance

$\sqrt{ }$ Yes/no question

Satisfaction

$\sqrt{ }$ Five-point scale

\section{Figures}




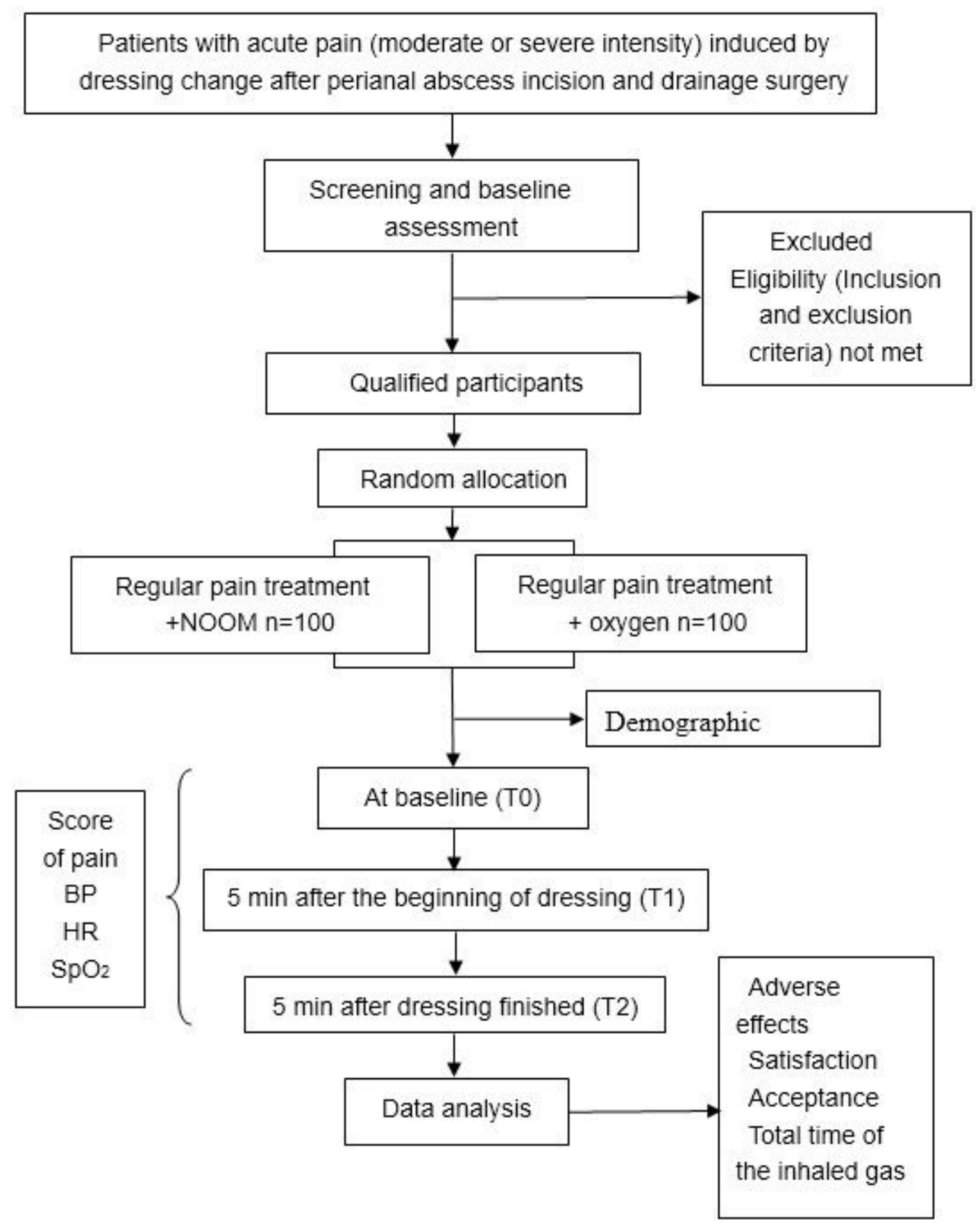

\section{Figure 1}

Research design roadmap. BP:blood pressure; HR:heart rate; Sp02:oxygen saturation; NOOM:nitrous oxide/oxygen. At baseline:3 min before dressing.

\section{Supplementary Files}

This is a list of supplementary files associated with this preprint. Click to download.

- SPIRITChecklist.doc 